\title{
Financial toxicity in cancer care
}

\author{
Jeremy M O'Connor, MD, ${ }^{a}$ Sheetal M Kircher, MD, ${ }^{\mathrm{b}}$ and Jonas A de Souza, MD ${ }^{\mathrm{c}}$
}

${ }^{a}$ Department of Medicine, The University of Chicago Medicine; 'bivision of Hematology-Oncology, Department of Medicine, Northwestern University Feinberg School of Medicine; and 'Section of Hematology/Oncology, Department of Medicine, The University of Chicago Medicine; Chicago, Illionois

The cost of cancer care is increasing, with important implications for the delivery of high-quality, patient-centered care. In the clinical setting, patients and physicians express a desire to discuss out-of-pocket costs. Nevertheless, both groups feel inadequately prepared to participate in these discussions, and perhaps not surprisingly, the integration of these discussions into clinical practice seems to be the exception rather than the rule. The resulting neglect of financial issues has the potential to cause unnecessary suffering for oncology patients. In this paper, we review the most relevant literature on financial toxicity in cancer care. In addition, we discuss potential predictors of financial toxicity, and the recent development of instruments to help clinicians and researchers quantify financial burden.

$\mathrm{T}$ he cost of cancer care in the United States is increasing rapidly, in both absolute and relative terms, with far-reaching consequences for patients, physicians, and policymakers. ${ }^{1,2}$ In 2005, the National Institutes of Health estimated that the annual cost of cancer care exceeded $\$ 200$ billion nationally, with oncologic prescriptions accounting for more than $40 \%$ of Medicare drug expenditures. ${ }^{1,2}$ Over the past 20 years, total patient out-of-pocket costs have steadily increased, and now account for about $20 \%$ of all health care spending. ${ }^{3}$ The factors driving that trend are numerous: an aging population, greater access to care, development of innovative and expensive new therapies, and overuse of existing treatments. ${ }^{4}$ In recent years, the amount of research focusing on the financial toxicity of cancer care has grown tremendously.

In this context, we performed a review of the medical literature on financial toxicity. A systematic search was performed using the PubMED database, with terms including, but not limited to: financial toxicity, cost of care, cancer, cost-effectiveness, and out-of-pocket cost. We evaluated over 200 abstracts, and selected the most relevant and timely citations. Our review begins with an introduction to recent trends in cancer costs, the potential effects of these costs on medication adherence, quality of life, and willingness to pay, and the difficult issue of patientphysician communication regarding costs of care. We conclude with a discussion of the emerging concept of financial toxicity, along with descriptions of several novel instruments designed to more accurately quantify financial distress.

\section{The cost of cancer care}

The complexity of health care spending in the United States arises from an economic environment in which patients spend not only their own personal resources, but also the pooled resources of others, in the form of third-party public or private payers. ${ }^{1}$ This multilayered payment structure protects individual consumers from the sometimes exorbitant costs of health care goods and services, but can also make it difficult for them to judge the relative economic value of various medical interventions.

Unfortunately, a system with extensive cost sharing may inherently incentivize the inefficient use of limited health care resources. At the same time, one would expect demand for interventions of only modest benefit to decline rapidly if out-of-pocket expenses were to increase. ${ }^{1}$ The history of cost sharing can be traced back to the landmark RAND Health Insurance Experiment (HIE), which suggested that cost sharing could decrease health care expenditures without significantly increasing the risk of adverse health outcomes. ${ }^{5}$ Subsequently, these findings have been cited by insurers to justify additional cost sharing between themselves and the patients they insure. $^{4}$

In contrast to the findings of the RAND study, there is substantial evidence that cost sharing may increase the risk of adverse outcomes, especially for selected groups of patients. One study estimated that for every $1 \%$ increase in insurance premiums, between 164,000 and 300,000 patients would be expected to lose their employer-sponsored insurance coverage. ${ }^{6}$ From an oncologic perspective, it has been presumed that such patients would be more likely to

Accepted for publication February 2, 2016. Correspondence: Jonas A de Souza, MD; jdesouza@medicine.bsd.uchicago. edu. Disclosures: The authors report no relevant conflicts of interest. JCSO 2016;14:101-106. (C2016 Frontline Medical Communications. doi: 10.12788/jcso.0239. 
experience poor clinical outcomes, with lower rates of cancer screening, later stage at cancer diagnosis, and higher cancer-specific mortality. ${ }^{7}$ Even patients with insurance can suffer from significant financial burden, potentially leading to delayed initiation of treatment, medication noncompliance, and personal bankruptcy., A national survey of patients declaring bankruptcy for medical reasons found that more than $75 \%$ of them reported being insured at the onset of their illness. ${ }^{10}$ Among this group of patients, cancer was the most costly diagnosis, with mean out-of-pocket expenditures exceeding $\$ 35,000 .{ }^{10}$

The prevalence of financial burden among cancer patients seems to be significant. Studies of cancer patients, specifically, have found varying levels of self-reported financial distress, ranging from 32\%-85\%.,11 In a longitudinal survey of patients with nonmetastatic breast cancer, a third of patients reported a decline in financial status in the period following diagnosis, with a significant minority reporting out-of-pocket costs that exceeded $\$ 5,000$ per year. ${ }^{12} \mathrm{~A}$ national survey of nonelderly adults found higher out-ofpocket burden (broadly defined as health-related spending greater than $20 \%$ of annual income) among cancer patients, compared with others with or without chronic medical conditions. ${ }^{13}$

In this context, the efficacy of patient-physician communication regarding financial issues has become increasingly important, with both the American Society of Clinical Oncology and the Institute of Medicine categorizing cost of care discussions as an essential component of high-quality care. ${ }^{8,14}$

\section{Medication nonadherence}

The medical consequences of financially toxic cancer care can be significant. In particular, there is ample data correlating higher out-of-pocket costs with medication nonadherence in cancer patients. In a single-center survey of 300 cancer patients, $16 \%$ of patients reported high or overwhelming financial distress, and $27 \%$ reported medication nonadherence because of financial concerns. ${ }^{15}$ Fiftytwo percent of those patients reported a desire to discuss out-of-pocket costs with their physician, whereas just 19\% reported having had these discussions. ${ }^{15}$ Patients who experienced higher-than-expected financial burden were especially likely to be nonadherent. ${ }^{15}$ These findings suggest that setting realistic financial expectations could decrease the risk of nonadherence. Medication nonadherence provides immediate cost-savings for the patient, but increases the risk of various adverse outcomes. In fact, an estimated $33 \%-69 \%$ of all hospital admissions have been attributed to nonadherence, with an annual price tag of up to $\$ 100$ billion. ${ }^{16}$

Additional data supporting the link between out-ofpocket financial burden and medication nonadherence comes from a retrospective cohort study of aromatase inhibitors in patients with breast cancer. ${ }^{17}$ In this study, copayments of more than $\$ 30$ a month were independently associated with nonpersistence, defined as a gap in prescription use of more than 45 days, among women older than 65 years. ${ }^{17}$ Findings from multiple studies have demonstrated an association between early discontinuation of aromatase inhibitors and worse overall survival in breast cancer patients. ${ }^{17}$ Similarly, chronic myeloid leukemia patients with higher copayments were almost twice as likely as those with lower copayments to discontinue treatment with tyrosine kinase inhibitors during the first 180 days of treatment ( $17 \%$ vs $10 \%$, respectively). ${ }^{18}$ Of course, financial concerns may not be the primary driver of medication nonadherence in these patients, and these studies may or may not have sufficiently controlled for other relevant variables.

In a more selected cohort of cancer patients applying for copayment assistance, $42 \%$ reported significant or catastrophic financial burden, and $20 \%$ of those patients reported taking less than the prescribed amount of medication for financial reasons. ${ }^{4}$ In this cohort, self-reported financial burden was similarly associated with several other coping behaviors, including reduced spending on leisure activities, selling possessions or property, and use of savings to pay for treatment-related expenses. ${ }^{4}$

Physicians have begun to recognize that out-of-pocket costs can contribute to unacceptable levels of financial distress among cancer patients. ${ }^{19}$ Unfortunately, discussing the financial implications of various treatment regimens can be exceedingly difficult. Inconsistent definitions of out-of-pocket costs, and difficulty predicting their magnitude, are 2 of the most frequently cited factors preventing effective cost of care communication. Some researchers use to the term "out-of-pocket" specifically to refer to the unreimbursed costs of treatment among insured individuals, whereas others include any therapy intended to prevent complications of treatment (such as administration of myeloid growth factors, blood transfusions, and antiemetics), along with costs of services meant to improve quality of life (such as acupuncture, home care, and physical therapy). Because costs for some or all of these services are unpredictably affected by treatment choice, insurance requirements, and pharmaceutical pricing, it may be impossible for physicians to accurately predict the costs of care for an individual patient. ${ }^{19}$ Understanding the difference between the total costs of the treatment and the total charges paid by the individual may be beyond the reasonable scope of practice for many providers. Similarly, it may be impossible for providers to accurately predict the cost-effectiveness of a treatment for any particular patient, because the benefits of treatment can be expected to vary widely among different individuals. In some cases, it may be preferable for providers to recommend more costly treatments when given several options with similar clinical efficacy, because 
of patient-specific concerns regarding side effects, convenience, or quality of life.

Despite these challenges, multiple authors have hypothesized that effective out-of-pocket cost discussions might facilitate the delivery of higher-quality patient care.${ }^{19}$ Cost discussions could help patients choose lower-cost treatment regimens, especially when faced with therapeutically equivalent alternatives. ${ }^{19}$ Furthermore, these discussions could allow patients to make purposeful concessions of therapeutic benefit to ensure lower personal financial burden. ${ }^{19}$ Finally, incorporating cost considerations into routine clinical decision-making could encourage more efficient delivery of quality care, which may have particular importance for more severely resource-limited settings. ${ }^{19}$

\section{Financial distress and quality of life}

There is increasingly strong evidence supporting the relationship between higher levels of financial distress and decreased quality of life. Among solid tumor patients undergoing active treatment, up to $47 \%$ report significant or catastrophic financial burden, with high levels of burden associated with low levels of patient satisfaction. ${ }^{20}$ Understanding this association may help identify interventions to improve patient satisfaction, which is increasingly being used as a metric for delivery of patient-centered care. ${ }^{20} \mathrm{It}$ is interesting to note that patient satisfaction with a physician's interpersonal manner, communication skills, and accessibility had no association with financial burden, suggesting that patients may continue to view individual physicians favorably despite significant financial burden. ${ }^{20}$ Fenn and colleagues, in a national survey of more than 2,000 cancer survivors, found that $30 \%$ of respondents reported some degree of cancer-related financial distress (in response to the question, "To what degree has cancer caused financial problems for you and your family?"). ${ }^{21}$ Most importantly, the authors found that the degree to which cancer caused financial problems, when compared with various other factors (including age, race, insurance status, and family income), was the strongest independent predictor of quality of life. ${ }^{21}$

In a study of cancer survivors, Zafar and colleagues found that high financial burden (defined as difficulty living on total household income) was associated with poorer quality of life, as measured by responses to the EuroQol group's self-reported measure, the EQ-5D.22 In a separate cohort of patients with advanced cancer, DelgadoGuay and colleagues found that financial distress was correlated with health-related quality of life (HR-QoL), as measured by the Functional Assessment of Chronic Illness Therapy-General (FACT-G; $r=0.23, P=.0057) .{ }^{23}$ Finally, de Souza and colleagues, using a financial toxicity patientreported outcome measure (PROM) ${ }^{24,25}$ termed COST (Comprehensive Score for Financial Toxicity), reported a statistically significant correlation between the patient's financial toxicity score and HR-QoL, as measured by the FACT-G $(\mathrm{r}=0.42, P<.001){ }^{26}$

\section{Willingness to pay}

The extent to which individual patients will pay for marginally better clinical outcomes has been examined. Johnson and colleagues conducted a discrete choice experiment to explore willingness to pay for prophylactic granulocyte colony-stimulating factor to decrease the risk of febrile neutropenia. ${ }^{27}$ In this study, patients were willing to pay more than $\$ 1,000$ to reduce the risk of disrupting their chemotherapy schedule, and more than $\$ 800$ to reduce the risk of infection. ${ }^{27}$ Among patients with non-Hodgkin lymphoma, a similar experiment found that for each $\$ 10$ increase in out-of-pocket costs, the adjusted odds ratio for accepting outpatient care for febrile neutropenia was $0.84 .{ }^{28} \mathrm{It}$ is interesting to note that the adjusted odds ratio for accepting outpatient care was even lower (0.53) for each $5 \%$ increase in probability of return to the hospital, suggesting that risk of hospitalization may be a greater deterrent than small increases in out-of-pocket costs. ${ }^{28}$

A study by Wong and colleagues similarly explored the complex decision-making of cancer patients faced with trade-offs between cost, efficacy, and toxicity. ${ }^{29}$ When presented with hypothetical treatment scenarios, patients with higher incomes were more likely to prioritize survival, whereas patients with lower incomes were more likely to prioritize avoidance of expensive treatments, regardless of survival or toxicity. The authors concluded that insurance plans with greater cost sharing may increase disparities in cancer care, specifically owing to cost-aversive behaviors among low-income patients. ${ }^{29}$ Willingness to pay for better overall survival is more difficult to assess systematically. An Australian study presented patients with a hypothetical scenario, asking whether they would be willing to pay $\$ 25,000$ out-of-pocket for an additional 4-6 months of survival. Among this patient population, about half of the patients (51\%) expressed a willingness to do so. ${ }^{30}$

\section{Patient-physician communication and cost of care}

Numerous potential obstacles can preclude effective costof-care discussions between patients and physicians. For patients, these barriers include uncertainty about the appropriateness of the topic, embarrassment, and the perception that a physician's time is limited. ${ }^{31}$ Some patients may hypothesize that voluntarily sharing stories of financial distress would compromise quality of care, by encouraging physicians to recommend less expensive, and potentially less effective, treatments. ${ }^{32,33}$ For physicians, potential barriers include a lack of knowledge about a patient's socioeconomic status, and uncertainty about a patient's desire to discuss the costs of care. ${ }^{31}$

A survey of medical oncologists found that most (80\%) 
thought it was important to be explicit about the potential financial impact of treatment choices. ${ }^{31}$ However, nearly 1 in 3 oncologists reported a high degree of discomfort about having these discussions with patients. Some oncologists avoid cost considerations entirely, focusing exclusively on treatments with maximal clinical benefit. ${ }^{31}$ A survey of breast cancer patients found that $94 \%$ of respondents thought physicians should discuss costs of care, whereas $14 \%$ reported having actually had such discussions. ${ }^{34}$ Another survey of cancer patients found that a majority (59\%) wanted physicians to discuss out-of-pocket costs with them. However, a similar percentage (57\%) reported that they did not integrate out-of-pocket costs into their individual medical decision-making process. ${ }^{35}$ These findings are supported by a smaller study of patients with localized prostate cancer, in which more than $90 \%$ of respondents, including patients with high financial burden, reported that they would not have chosen a different treatment, even if they had prior knowledge of out-of-pocket costs. ${ }^{36}$ Consequently, the desire to discuss treatment costs should not be assumed to represent a simultaneous desire to integrate cost-saving behaviors into clinical decision making. ${ }^{35}$ In a survey of breast cancer patients, almost all (96\%) wanted to discuss expensive drug options, even if they were unlikely to be affordable. ${ }^{25}$ Similar data were found in a study among Australian cancer patients, with more than $90 \%$ of patients expressing a desire to learn about prohibitively expensive cancer treatments. ${ }^{30}$

The integration of midlevel providers, social workers, and registered nurses into cost-of-care discussions may facilitate more timely and effective communication. Unfortunately, there are very few published studies of cost communication practices among nonphysician providers. A single-center qualitative survey reported frustration among social workers about the lack of financial resources for patients, institutional barriers, and limited resources for identification of at-risk patients. ${ }^{37}$

\section{Financial toxicity: an emerging concept}

The terms financial distress, financial burden, and financial toxicity have been used interchangeably to describe the financial impact of cancer care. ${ }^{9,38}$ Financial toxicity, in particular, was first referenced in 2009 to underscore the dramatic economic effects of modern oncology drugs, and has since been adopted by multiple authors. ${ }^{4,24,39,40}$ This term emphasizes the clinical relevance of financial distress, as an equivalent to the more widely acknowledged physical and psychological toxicities of cancer treatment.

Identifying patients who are at highest risk of financial toxicity has been challenging. In a study of patients with colorectal cancer, the presence of postsurgical complications was associated with higher levels of financial burden and higher self-reported financial worry. ${ }^{41} \mathrm{~A}$ study from the pediatric oncology literature found that younger, less educated parents were more likely to report financial hardship. ${ }^{42}$ In another study, a correlation was found between unexpected hospitalizations, employment disruptions, and reported financial burden among primary caretakers of children with cancer. ${ }^{43}$ Older studies have suggested that financial toxicity peaks immediately after the time of diagnosis, when admissions are frequent, work disruptions are ubiquitous, and insurance benefits have yet to be distributed. ${ }^{44,45}$

Among adult patients, both younger age and larger household size have been associated with financial toxicity. ${ }^{4}$ Fenn and colleagues found that patients reporting "a lot" of financial distress were more likely to be nonwhite, female, and younger than 61 years old, with a total annual household income of less than $\$ 35,000$, and less than a college degree. ${ }^{21}$ Among patients with nonmetastatic breast cancer, underserved patients were most vulnerable to financial decline, after adjusting for income, educational level, and employment status. ${ }^{12}$ Among patients with stage III colorectal cancer receiving adjuvant chemotherapy, the 2 factors most strongly associated with financial hardship were younger age and lower annual household income. ${ }^{46}$ In a cohort of patients with solid tumors seeking financial assistance, use of cost-coping strategies was associated with younger age, lower income, higher education, and shorter duration of chemotherapy. ${ }^{47}$ Younger patients may have more difficulty than older individuals in adjusting to the financial pressures of cancer care for many reasons, including higher baseline household expenses and less time to accumulate necessary financial assets. ${ }^{46}$ In a recent study, Huntington and colleagues used the COST instrument to assess 100 insured patients with multiple myeloma. ${ }^{48} \mathrm{In}$ that population, younger age $(P=.0092)$, lower household income $(P=.0031)$, nonmarried status $(P=.0074)$, and longer time since diagnosis $(P=.042)$ were associated with greater financial toxicity.

It is certainly possibly, if not likely, that different diseases have different drivers of financial toxicity. Therefore, the adoption of a validated tool for the measurement of financial toxicity, encompassing both out-of-pocket costs and loss-of-income concerns, is necessary to facilitate accurate comparisons of financial toxicity among different populations. For these reasons, de Souza and colleagues developed the COST Patient-Reported Outcome Measure, using standardized statistical methods, to more accurately quantify financial toxicity among different cohorts of cancer patients. ${ }^{24,28}$ In the development study performed in 155 patients, the single-factor solution explained 93\% of the variance in the data, with communalities between 0.3 and 0.7. The Cronbach alpha coefficient for the 11-item COST measure was 0.9 , indicating excellent internal consistency. Mean inter-item and item-total correlations were 0.47 (range, 0.22-0.69) and 0.71 (range, 0.62-0.79), respectively, demonstrating nonredundancy and good construct 
validity. ${ }^{24}$ In a second cohort of 233 patients with advanced cancers, the authors demonstrated a significant relationship between financial toxicity and younger age $(P<.01)$, nonwhite ethnicity $(P<.05)$, less than a college degree $(P$ $<.01)$, unemployment $(P<.001)$, Medicaid insurance $(P$ $<.05)$, and lower income $(P<.001) .{ }^{26}$ In addition, subsequent data from this cohort clearly demonstrated a graded relationship between financial toxicity (as measured by the COST instrument) and health-related quality of life. ${ }^{26}$

Adapting questions from the National Consumer Bankruptcy Project, Veenstra and colleagues developed a similar patient-reported outcome measure for patients with a current or prior history of stage III colorectal cancer. ${ }^{49}$ The composite measure was internally validated within the same sample against a binary question (My illness has had no impact on my finances) and a single question about financial worry (How much do you worry about financial problems that have resulted from your colorectal cancer and its treatment?). Among the 956 colorectal cancer patients, factor analysis of 7 burden items yielded a single-factor solution, with all factor loadings greater than 0.4. Internal consistency as measured by the Cronbach alpha was $0.79 .{ }^{49}$

Another commonly used instrument for quantifying financial distress is the Financial Well-Being Scale, a 10 -item survey to evaluate the general population's reactions to their financial situation. ${ }^{50}$ Other studies have used single-item questionnaires to associate financial problems with various quality of life measures. ${ }^{22,23}$ The decision

\section{References}

1. Meropol NJ, Schulman KA. Cost of cancer care: issues and implications. J Clin Oncol. 2007;25:180-186.

2. American Cancer Society. Cancer facts \& figures 2006. http://www. cancer.org/research/cancerfactsfigures/cancerfactsfigures/cancerfacts-figures-2006. Published 2006. Accessed February 10, 2016.

3. Alexander GC, Casalino LP, Meltzer DO. Patient-physician communication about out-of-pocket costs. JAMA. 2003;290:953-958.

4. Zafar SY, Peppercorn JM, Schrag D, et al. The financial toxicity of cancer treatment: a pilot study assessing out-of-pocket expenses and the insured cancer patient's experience. Oncologist. 2013;18:381-390.

5. Brook RH, Ware JE, Rogers WH, et al. Does Free Care Improve Adults' Health? New England Journal of Medicine. 1983;309(23):1426-1434.

6. Chernew M, Cutler DM, Keenan PS. Increasing health insurance costs and the decline in insurance coverage. Health Serv Res. 2005;40:1021-1039.

7. Ward E, Halpern M, Schrag N, et al. Association of insurance with cancer care utilization and outcomes. CA Cancer J Clin. 2008;58:9-31.

8. Meropol NJ, Schrag D, Smith TJ, et al. American Society of Clinical Oncology guidance statement: the cost of cancer care. J Clin Oncol. 2009;27:3868-3874.

9. de Souza J, Wong Y-N. Financial distress in cancer patients. J Med Pers. 2013;11:73-77.

10. Himmelstein DU, Warren E, Thorne D, Woolhandler S. Illness and injury as contributors to bankruptcy. http://content.healthaffairs.org/content/suppl/2005/01/28/hlthaff.w5.63.DC1. Health Aff. Published February 2005. Accessed February 10, 2016.

11. Sharp L CA, Timmons A. Associations between cancer-related regarding which instrument to use should be determined by the emerging body of literature supporting their development and validation, as well as their association with clinically meaningful outcomes. Future integration of these instruments into routine clinical practice may promote earlier identification of patients at risk for financial toxicity, and ensure more transparent and standardized evaluations of financial health. ${ }^{33}$

\section{Conclusions}

The financial burden of cancer care in the United States is a growing problem for all stakeholders, including patients, providers, and third-party payers, with significant medical, psychological, and behavioral consequences. The current medical literature provides consistent evidence that financial toxicity can affect clinically relevant outcomes, like medication adherence and quality of life. Multiple authors have expressed the opinion that physicians should take a leading role in promoting cost-effective cancer care, and clearly communicate with patients regarding potential outof-pocket costs. As a consequence of recent national health care reforms in the United States, many experts expect to see a trend toward lower out-of-pocket costs, especially among the previously uninsured..$^{13}$ Nevertheless, in an era of ongoing and potentially unsustainable increases in the cost of care, financial toxicity should be expected to emerge as an increasingly relevant clinical problem, with far-reaching implications for the delivery of high quality, patientcentered cancer care.

financial stress and strain and psychological well-being among individuals living with cancer. Psychooncol. 2012;22:745-755.

12. Jagsi R, Pottow JA, Griffith KA, et al. Long-term financial burden of breast cancer: experiences of a diverse cohort of survivors identified through population-based registries. J Clin Oncol. 2014;32:1269-1276.

13. Bernard DS, Farr SL, Fang Z. National estimates of out-of-pocket health care expenditure burdens among nonelderly adults with cancer: 2001 to 2008. J Clin Oncol. 2011;29:2821-2826.

14. Ganz PAL, Laura A. Charting a new course for the delivery of highquality cancer care. J Clin Oncol. 2013;31:4485-4487.

15. Bestvina CM, Zullig LL, Rushing C, et al. Patient-oncologist cost communication, financial distress, and medication aherence. J Oncol Pract. 2014;10:162-168.

16. Osterberg L, Blaschke T. Adherence to medication. New Engl J Med. 2005;353:487-497.

17. Neugut AI, Subar M, Wilde ET, et al. Association between prescription co-payment amount and compliance with adjuvant hormonal therapy in women with early-stage breast cancer. J Clin Oncol. 2011;29:2534-2542.

18. Dusetzina SB, Winn AN, Abel GA, Huskamp HA, Keating NL. Cost sharing and adherence to tyrosine kinase inhibitors for patients with chronic myeloid leukemia. J Clin Oncol. 2014;32:306-311.

19. Ubel PA, Abernethy AP, Zafar SY. Full disclosure - out-of-pocket costs as side effects. New Engl J Med. 2013;369:1484-1486.

20. Chino F, Peppercorn J, Taylor DH Jr, et al. Self-reported financial burden and satisfaction with care among patients with cancer. Oncologist. 2014;19:414-420.

21. Fenn KM, Evans SB, McCorkle R, et al. Impact of financial burden 
of cancer on survivors' quality of life. J Oncol Pract. 2014;10:332-338.

22. Zafar SY, McNeil RB, Thomas CM, Lathan CS, Ayanian JZ, Provenzale D. Population-based assessment of cancer survivors' financial burden and quality of life: a prospective cohort study. J Oncol Pract. 2015;11:145-150.

23. Delgado-Guay M, Ferrer J, Rieber AG, et al. Financial distress and its associations with physical and emotional symptoms and quality of life among advanced cancer patients. Oncologist. 2015;20:1092-1098

24. de Souza JA, Yap BJ, Hlubocky FJ, et al. The development of a financial toxicity patient-reported outcome in cancer: the COST measure. Cancer. 2014;120:3245-3253.

25. Kaser E, Shaw J, Marven M, Swinburne L, Boyle F. Communication about high-cost drugs in oncology -the patient view. Ann Oncol. 2010;21:1910-1914.

26. de Souza JA, Wroblewski B, Yap BJ, et al. Grading financial toxicity based upon its impact on health-related quality of life (HRQoL). J Clin Oncol. 2015;33:Suppl; Abstr 6618.

27. Johnson P, Bancroft T, Barron R, et al. Discrete choice experiment to estimate breast cancer patients' preferences and willingness to pay for prophylactic granulocyte colony-stimulating factors. Value Health. 2014;17:380-389.

28. Lathia N, Isogai PK, Walker SE, et al. Eliciting patients' preferences for outpatient treatment of febrile neutropenia: a discrete choice experiment. Support Care Cancer. 2013;21:245-251.

29. Wong YN, Egleston BL, Sachdeva K, et al. Cancer patients' tradeoffs among efficacy, toxicity, and out-of-pocket cost in the curative and noncurative setting. Med Care. 2013;51:838-845.

30. Mileshkin L, Schofield PE, Jefford M, et al. To tell or not to tell: the community wants to know about expensive anticancer drugs as a potential treatment option. J Clin Oncol. 2009;27:5830-5837.

31. Schrag D, Hanger M. Medical oncologists' views on communicating with patients about chemotherapy costs: a pilot survey. J Clin Oncol. 2007;25:233-237.

32. Hofstatter EW. Understanding patient perspectives on communication about the cost of cancer care: a review of the literature. J Oncol Pract. 2010;6:188-192.

33. Shankaran V, Ramsey, S. Addressing the financial burden of cancer treatment: from copay to can't pay. JAMA Oncol. 2015;1:273-274.

34. Irwin B, Kimmick G, Altomare I, et al. Patient experience and attitudes toward addressing the cost of breast cancer care. Oncologist. 2014;19:1135-1140.

35. Bullock AJ, Hofstatter EW, Yushak ML, Buss MK. Understanding patients' attitudes toward communication about the cost of cancer care. J Oncol Pract. 2012;8:e50-58.

36. Jung OS, Guzzo T, Lee D, et al. Out-of-pocket expenses and treatment choice for men with prostate cancer. Urology. 2012;80:1252-1257.

37. Smith SK, Nicolla J, Zafar SY. Bridging the gap between financial distress and available resources for patients with cancer: a qualitative study. J Oncol Pract. 2014;10:e368-e372.

38. de Souza JA, Yap B, Ratain MJ, Daugherty C. User beware: we need more science and less art when measuring financial toxicity in oncology. J Clin Oncol. 2015;33:1414-1415.

39. Ratain MJ. Biomarkers and clinical care. Presented at the AAASFDLI Colloquium: personalized medicine in an era of health care reform. Washington, DC; October 27, 2009.

40. Zafar SY, Abernethy AP. Financial toxicity, Part II: how can we help with the burden of treatment-related costs? Oncology (Williston Park, NY). 2013;27:253-254,256

41. Regenbogen SE, Veenstra CM, Hawley ST, et al. The personal financial burden of complications after colorectal cancer surgery. Cancer. 2014;120:3074-3081.

42. Dussel V, Bona K, Heath JA, Hilden JM, Weeks JC, Wolfe J. Unmeasured costs of a child's death: perceived financial burden, work disruptions, and economic coping strategies used by American and Australian families who lost children to cancer. J Clin Oncol. 2011;29:1007-1013.

43. Warner EL, Kirchhoff AC, Nam GE, Fluchel M. Financial burden of pediatric cancer for patients and their families [published online ahead of print October 14, 2014]. J Oncol Pract. pii: JOP.2014.001495.

44. Bloom BS, Knorr RS, Evans AE. The epidemiology of disease expenses. The costs of caring for children with cancer. JAMA. 1985;253:2393-2397.

45. Eiser C, Upton P. Costs of caring for a child with cancer: a questionnaire survey. Child Care Health Dev. 2007;33:455-459.

46. Shankaran V, Jolly S, Blough D, Ramsey SD. Risk factors for financial hardship in patients receiving adjuvant chemotherapy for colon cancer: a population-based exploratory analysis. J Clin Oncol. 2012;30:1606-1614.

47. Nipp RD, Zullig LL, Samsa G, et al. Identifying cancer patients who alter care or lifestyle due to treatment-related financial distress [published online ahead of print July 7, 2015]. Psychooncology. doi: 10.1002/pon.3911.

48. Huntington SF, Weiss BM, Vogl DT, et al. Financial toxicity in insured patients with multiple myeloma: a cross-sectional pilot study [published online September 16, 2015]. Lancet Haematol. DOI: http://dx.doi.org/10.1016/S2352-3026(15)00151-9. Accessed February 10, 2016.

49. Veenstra CM, Regenbogen SE, Hawley ST, et al. A composite measure of personal financial burden among patients with Stage III colorectal cancer. Medical Care. 2014;52:957-962.

50. Prawitz AD, Garmen ET, Sorhaindo B, O’Neill B, Kim J, Drentea P. InCharge Financial Distress/Financial Well-Being Scale: development, administration, and score interpretation. Financial Counseling and Planning. 2006;17:34-50. 